$$
\begin{gathered}
N>N_{\epsilon} \text { and } \lambda_{\epsilon}<\lambda<1, \\
\left|D\left(x_{0}\right)\right| \leqq \sum_{j=0}^{\infty} I_{j}<\epsilon,
\end{gathered}
$$

by the above inequalities and (4.1) of the paper cited in footnote 1 . The same corollary as in that paper holds now for the functional of (1)-and for the same reasons as given in the proof for the bounded functional.

The University of Minnesota

\title{
ESSENTIALLY ADMISSIBLE SEQUENCES
}

\section{R. CREIGHTON BUCK}

Let $X$ be the set of all complex sequences $\alpha=\left\{a_{n}\right\}$ such that $\|\alpha\|=\sup _{n}\left|a_{n}\right|^{1 /(n+1)}<\infty$. Under the usual operations, $X$ is a complex vector space, and $\|\alpha+\beta\| \leqq\|\alpha\|+\|\beta\|$. However, $\|c \alpha\|$ is seldom $|c|\|\alpha\|$ and even though $c_{n} \rightarrow 0$, it is not in general true that $\left\|c_{n} \alpha\right\| \rightarrow 0$; for example, if $\alpha=\langle 1,1,1, \cdots\rangle$, then $\|c \alpha\|$ is $c$ if $c \geqq 1$ and is 1 if $0<c<1$. Defining the distance between $\alpha$ and $\beta$ as $\|\alpha-\beta\|, X$ becomes a complete metric abelian group, but not a topological linear space. If with each $\alpha$ in $X$ is associated the analytic function defined at the origin by $f(z)=\sum a_{n} z^{n}$, then this topology is that in which a sequence $\left\{f_{n}\right\}$ converges to the zero function only if on every bounded domain $D$, and for sufficiently large $n$, the functions $f_{n}$ are all analytic on $D$ and converge uniformly to zero; $f_{n}$ converges to $g$ if $f_{n}-g$ converges to zero. This topology is closely related to that introduced by Ganapathy Iyer into the vector space of all entire functions [4].

Given any $\alpha \in X$, there may be found an entire function $f(z)$ of order 1 , finite type, and such that $f(i y)=O(\exp c|y|)$ for some $c \leqq \pi$, which interpolates to $\alpha$ in the sense that $f(n)=a_{n}$ for $n=0,1,2, \ldots$ [1]. This is not the case if the condition $c<\pi$ is imposed. We have called a sequence $\alpha$ admissible in case such a more restricted function exists [2]. By a theorem of Carlson, such a function when it exists is unique [3]. A sequence $\alpha$ may fail to be admissible in an inessential way; for example, $\alpha=\langle 0,0,0, \ldots\rangle$ is admissible, but $\beta$ $=\langle 1,0,0, \cdots\rangle$ is not. To allow for this, we shall now say that $\alpha$

Presented to the Society, September 4, 1952; received by the editors October 16, 1952. 
is essentially admissible if there is a function $f(z)$ with $c<\pi$ such that $f(n)=a_{n}$ for all sufficiently large values of $n$. The set of all such $\alpha$ forms a subset of $X$ which we denote by $A$. Because of the theorem of Carlson cited above, one expects $A$ to be a "sparse" subset of $X$. The object of this note is to give this conjecture a precise form. We first observe that if $\alpha$ and $\beta$ lie in $A$, so does $\alpha+\beta$, and $c \alpha$ for any complex $c$. Moreover, if $\alpha^{\prime}$ is obtained from $\alpha$ by altering any finite set of its coordinates, $\alpha^{\prime}$ is also in $A$. The study of $A$ may be confined to that portion of it lying in the open unit sphere, since if $\|\alpha\|=R$ and $\alpha \in A$, the sequence $b_{n}=(1 / 2 R)^{n+1} a_{n}$ is also in $A$, and $\|\beta\|=1 / 2$.

Theorem 1. $A$ is of first category in $X$.

In spite of this, it might be supposed that $A$ is dense in $X$. This is not the case.

Theorem 2. $A$ is not dense in $X$. In fact, there are open subsets of the unit sphere which contain no points of $A$.

It will be seen that there are therefore spheres of arbitrarily large size (radius) which are free of points of $A$. We conjecture that $A$ itself is nondense.

Before proving these, we introduce certain definitions. For any $a$ and $c$, let $K(a, c)$ be the set of those entire functions $f(z)$ obeying $f(z)=O(1)(\exp a|x|+c|y|)$, and let $A(a, c)$ be the corresponding subset of $A$, comprising those sequences $\alpha$ interpolated to by functions in $K(a, c)$. We have $A=\bigcup A(a, c)$ if the union is taken for all $0 \leqq a<\infty$ and $0 \leqq c<\pi$. Let $A^{*}$ be the union of the closures of the sets $A(a, c)$. This of course may be only part of the closure of $A$.

Given any $\alpha \in X$, let $b_{n}=\Delta^{n} a_{0}=(-1)^{n} \sum C_{n, k}(-1)^{k} a_{k}$ and $g(z)$ $=\sum b_{n} z^{n}$. We shall make use of a simple identity connecting $g(z)$ and the function $F(z)=\sum a_{n} z^{n}$. (See for example [5].)

$$
(1+z) g(z)=F(z /(1+z)), \quad(1-z) F(z)=g(z /(1-z)) .
$$

The basis for our proofs for the theorems stated above is the following characterization of the set $A^{*}$.

THEOREM 3. $\alpha \in A^{*}$ if and only if $g(z)$ is regular at the origin and has an extension to a neighborhood of $-1 \leqq x \leqq 0$ except possibly for an isolated singularity at -1 .

This depends upon the following characterization for the smaller class of admissible sequences [2]: $\alpha$ is admissible if and only if $g(z)$ is regular on a neighborhood of the interval $-1 \leqq x \leqq 0$. This neighborhood contains an open set $\Omega(a, c)$ depending only on the growth 
constants $a, c$, of the entire function $f(z)$ which interpolates to $\alpha$. Let $\alpha$ lie in the set $A(a, c)$. Choose an admissible $\beta=\left\{b_{n}\right\}$ such that $c_{n}=a_{n}-b_{n}=0$ for all $n>n_{0}$. We have

$$
g(z)=\sum \Delta^{n} b_{0} z^{n}+\sum \Delta^{n} c_{0} z^{n}=g_{1}(z)+g_{2}(z) .
$$

Since $\beta$ is admissible, $g_{1}$ is regular on $\Omega(a, c)$ containing $-1 \leqq x \leqq 0$. Since $c_{n}=0$ for $n>n_{0}, F_{2}(z)=\sum c_{n} z^{n}$ is a polynomial, and, using (1), $g_{2}(z)$ has a pole at -1 as its only singularity. $g(z)$ is then regular in $\Omega(a, c)$ except for a pole at -1 . Conversely, if this is true, $g(z)$ may be written as $g_{1}(z)+P(z) /(1+z)^{m}$ where $P$ is a polynomial, and $a_{n}=b_{n}+c_{n}$ where $\beta=\left\{b_{n}\right\}$ is admissible, and $c_{n}=0$ for all large $n$. Let us now suppose that $\alpha$ is a limit point of $A(a, c)$. Given $\epsilon>0$, choose $\beta \in A(a, c)$ with $\|\alpha-\beta\|<\epsilon<1$, and let $\left\{c_{n}\right\}=\alpha-\beta$. Then $g(z)$ $=\sum \Delta^{n} b_{0} z+\sum \Delta^{n} c_{0} z^{n}=g_{1}(z)+g_{2}(z) . g_{1}(z)$ is regular in $\Omega(a, c)$ except for a possible pole at -1 . Since $\left|c_{n}\right|<\epsilon^{n+1}$ for all $n, \sum c_{n} z^{n}$ is regular for $|z|<1 / \epsilon$ and, by (1), $g_{2}(z)$ is regular outside the disc $|z /(1+z)|$ $\geqq 1 / \epsilon . g(z)$ is then regular on the set obtained by deleting this disc from $\Omega(a, c)$. Letting $\epsilon$ decrease, $g(z)$ is regular on all of $\Omega(a, c)$ except possibly at -1 . Conversely, let $g(z)$ be regular on a neighborhood $\Omega$ of $[-1,0]$ except for an isolated singularity at -1 . Write $g(z)$ $=g_{1}(z)+g_{2}(z)=\sum \Delta^{n} b_{0} z^{n}+\sum \Delta^{n} c_{0} z^{n}$ where $g_{1}$ is regular in $\Omega$, and $g_{2}$ has its only singularity at -1 . Putting $\beta=\left\{b_{n}\right\}$, we see by the result cited above that $\beta$ is admissible, and hence in $A(a, c)$ for a suitable choice of $a$ and $c$. By (1), $\sum c_{n} z^{n}$ is entire, and $\lim \left|c_{n}\right|^{1 / n}=0$. Setting $\gamma=\left\{c_{n}\right\}$, we have $\alpha=\beta+\gamma$, with $\beta \in A(a, c)$. It is not necessarily true that $\|\gamma\|$ is small. However, by a slight shift, we can show that $\alpha$ lies in the closure of $A(a, c)$. For any $N$, set

$$
c_{n}^{\prime}=\left\{\begin{array}{ll}
0, & n \leqq N, \\
c_{n}, & n>N,
\end{array} \quad b_{n}^{\prime}= \begin{cases}b_{n}+c_{n}, & n \leqq N, \\
b_{n}, & n>N .\end{cases}\right.
$$

Then, $\alpha=\beta^{\prime}+\gamma^{\prime}$, and $\left\|\alpha-\beta^{\prime}\right\|=\left\|\gamma^{\prime}\right\|=\sup _{n \geqq N}\left|c_{n}\right|^{1 /(n+1)}$, which may be made arbitrarily small by increasing $N$. Since $\beta^{\prime}$ agrees with $\beta$ at all but a finite number of coordinates, $\beta^{\prime}$ lies in $A(a, c)$.

We next proceed to the proof of Theorem 1 and Theorem 2 .

Proof of Theorem 1. Since $A=\bigcup_{n, m} A(n, \pi-1 / m)$, we shall show that $A$ is of first category if we show that each $A(a, c)$ is nondense. Given $\beta \in A(a, c)$ and $\epsilon>0$, we shall produce $\alpha$ such that $\|\beta-\alpha\|<\epsilon$, but such that $\alpha$ is not in the closure of $A(a, c)$. Let $\gamma=\left\{c_{n}\right\}$ where $c_{n}$ is 0 if $n$ is a square, and $\epsilon^{n+1}$ otherwise, and set $\alpha=\beta+\gamma$. Clearly, $\|\gamma\|=\epsilon$. The function $\sum c_{n} z^{n}=\sum \epsilon^{k^{2}+1} z^{k^{2}}$ has the circle $|z|=1 / \epsilon$ as a cut. By (1), $g_{2}(z)=\sum \Delta^{n} c_{0} z^{n}$ is regular for $\epsilon|z|<|1+z|$, and the boundary of this is a cut. Since $\beta$ is in $A(a, c), g_{1}(z)=\sum \Delta^{n} b_{0} z^{n}$ is 
regular on a neighborhood of $-1 \leqq x \leqq 0$, and $g(z)=g_{1}(z)+g_{2}(z)$ does not have the type of behavior which permits $\alpha$ to be in the set $A^{*}$.

Proof of Theorem 2. We must show that there are open sets in $X$ which contain no points of $A$. For this we use a special theorem concerning oscillating sequences: if $C_{1}$ and $C_{2}$ are two disjoint convex sets and if the terms of the sequence $\alpha$ alternate between these sets, $\alpha$ is not admissible [1]. Let $c>1$ and consider the special sequence $\beta=\left\{b_{n}\right\}$ where $b_{n}=c(-1)^{n}$. If, now, $\|\alpha-\beta\| \leqq 1$, then $\left|a_{n}-c(-1)^{n}\right|$ $\leqq 1$ for all $n$, so that $a_{n}$ alternates between the circles of unit radius with center at $c$ and $-c$. Thus, the sphere, center $\beta$ and radius 1 , is disjoint from $A$. More generally, if $b_{n}=(-1)^{n} R^{n+1}$ then the open sphere, center $\beta$ and radius $R$, is disjoint from $A$. It should be noted that $\|\beta\|=R$, so that these $A$-free spheres can be found at any distance from the origin.

\section{REFERENCES}

1. R. C. Buck, $A$ class of entire functions, Duke Math. J. vol. 13 (1946) pp. 541559.

2. - Admissible sequences, and a theorem of Polya, Comment. Math. Helv. vol. 27 (1953) pp. 75-80.

3. F. Carlson, Sur une classe de series de Taylor, Thesis, Uppsala.

4. Ganapathy Iyer, The space of integral functions (I), J. Indian Math. Soc. N.S. vol. 12 (1948) pp. 13-80.

5. H. Pollard, Sequences with vanishing even differences, Duke Math. J. vol. 12 (1945) pp. 303-304.

UNIVERSITY OF WISCONSIN 